\title{
A Rare Case of Acute Necrotizing Gastritis
}

\author{
Ravi Kumar Sabu Murugesan ${ }^{1} \cdot$ Suganth Annamalai ${ }^{1} \cdot$ Joyce Prabakar ${ }^{1} \cdot$ Kannan Ross $^{1}$
}

Received: 10 March 2021 / Accepted: 7 May 2021 / Published online: 17 May 2021

(C) Association of Surgeons of India 2021

\begin{abstract}
Stomach is a highly vascular organ in the gastrointestinal tract. It is very rare for a stomach to go in for gangrene even in cases of volvulus. Spontaneous gangrene due to acute necrotizing gastritis is a very rare and dreaded condition. This condition is usually not recognized preoperatively due to its rarity. Hence, early diagnosis and prompt expert management are necessary. Here, we present a case of acute necrotizing gastritis which was admitted in our emergency department which was diagnosed to be a case of gastric gangrene preoperatively with the help of radiological investigations.
\end{abstract}

Keywords Gastric gangrene $\cdot$ Stomach $\cdot$ Necrotizing gastritis $\cdot$ Shock

\section{Introduction}

Acute necrotizing gastritis is a variant of phlegmonous gastritis, with organisms producing necrosis and gangrene of the stomach wall rather than just an intramural abscess [1]. In acute necrotizing gastritis, all four major gastric vessels are patent, but gastric gangrene occurs secondary to an overwhelming necrobiotic infection [2]. Here, we report a case of 30-year-old gentleman without any previous complaints and comorbidities presented with acute necrotizing gastritis.

\section{Case Report}

A 30-year-old gentleman presented with features of abdominal pain, fever, and vomiting for the past 2 days. He was on intermittent long duration fasting for the past 15 days. Pain was severe in the epigastric region which was non-radiating and was aggravated by food intake. He had normal bowel movements. On examination, patient was anxious, oriented, febrile with temperature of $101^{\circ} \mathrm{F}$. There was no pallor, icterus. His vitals recorded a pulse rate of 120 per minute, blood pressure 90/60 $\mathrm{mmHg}$, and normal oxygen saturation. His per abdomen findings revealed diffuse abdominal tenderness, more in the epigastric region which was associated with

Ravi Kumar Sabu Murugesan

mrksabu65@gmail.com

1 Institute of General Surgery, Madras Medical College, Chennai Tamil Nadu India guarding. There was upper abdominal distension. There was no audible bowel sounds. Per rectal examination revealed foul smelling blood tinged discharge. Patient was immediately resuscitated with crystalloids, antibiotics and analgesics. Nasogastric tube was inserted through which $2 \mathrm{~L}$ collection of thick foul smelling, blood tinged fluid was drained. Complete blood count revealed leukocytosis. Patient was immediately shifted for radiological investigations. Contrast enhanced computed tomography findings revealed grossly distended stomach with air fluid levels (Fig. 1). Prominent intramural gas noted along the greater and lesser curvature along with reduced enhancement of proximal portion of gastric wall (Fig. 2). Multiple air pockets tracking to the portal venous system are noted. One peculiar feature seen was reduced aortomesentric angle (19 degree) which made a confusion in the diagnosis.

Free fluid noted in the abdomen and pelvis. Patient was proceeded with laparotomy. Intraoperative findings revealed frank gangrene of the stomach involving the greater curvature and fundus. The lesser curvature, gastroduodenal junction, and the gastroesophageal junction were spared. There was no evidence of atherosclerosis, volvulus, or herniation. Duodenum looked normal. Small bowel was dilated and mildly edematous (Fig. 3).

Total gastrectomy was performed, and duodenal stump closed in two layers, planned for esophagojejunostomy (Fig. 4). In view of intraoperative hypotension, patient was started on dual inotrope supports. The distal esophageal end was friable. In view of the above 2 factors, closure of distal esophageal end with drain, feeding jejunostomy, and cervical esophagostomy was done. 
Fig. 1 Shows grossly distended stomach with intramural gas

Fig. 2 Shows portal venous air the right upper image
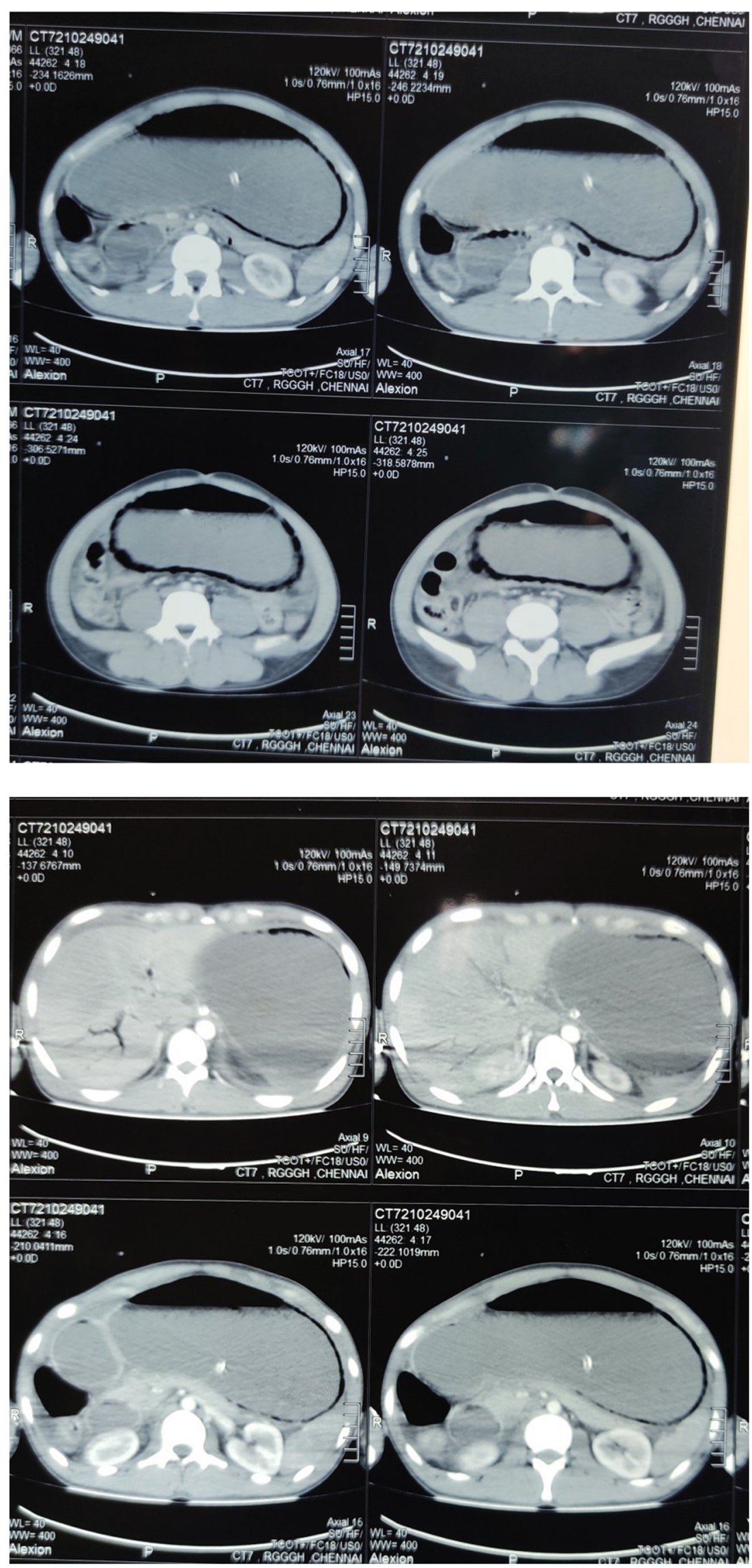


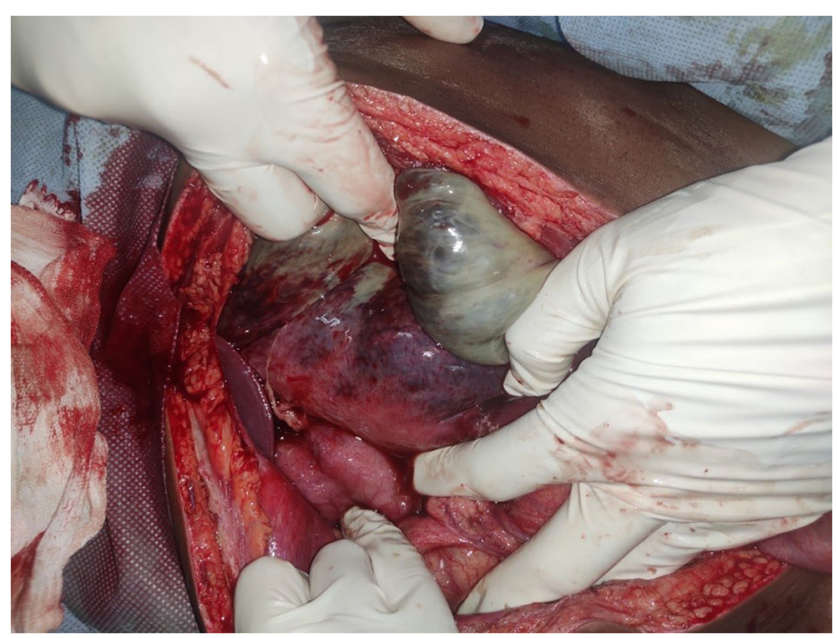

Fig. 3 Intraoperative picture showing transition zone between the viable antrum and the necrotic stomach

Patient could not be extubated on table due to severe hemodynamic instability and severe metabolic acidosis and was on ventilator support in the surgical intensive care unit. Inspite of maximum dose of inotrope drug and acidosis correction, patient's hemodynamic instability persisted, and he expired on the first postoperative day.

Discussion The stomach is a well-vascularized organ that receives a blood supply from the left gastric artery (a branch of celiac axis), the right gastric artery (a branch of the common hepatic artery), the right gastroepiploic artery (a branch of the gastroduodenal artery), the left gastroepiploic artery (a branch of the splenic artery), and the short gastric arteries, which also arise from the splenic artery. Therefore, gastric infarction is

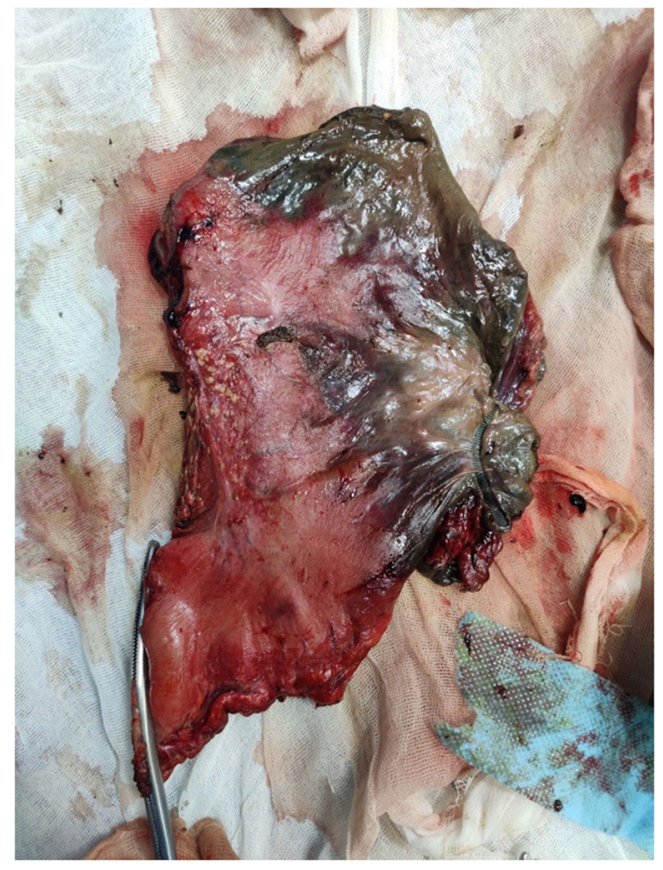

Fig. 4 Shows resected total gastrectomy specimen uncommon compared with other parts of the gastrointestinal tract [3]. Gastric gangrene could be secondary to atherosclerosis, arterial embolism, iatrogenic gel foam embolism, venous thrombosis, gastric volvulus, bulimia nervosa, endoscopic hemostatic injections, diaphragmatic hernia, and infectious gastritis [4]. The pathogenesis is unclear, although predisposing factors include chronic gastritis, increased age, alcoholism, hypoacidity, protein-energy malnutrition, and immunosuppression [5]. In acute gastric ischemia, patients can develop nausea, vomiting, upper GI bleeding, abdominal distention, and symptoms and signs related to underlying predisposing conditions [6]. Phlegmonous gastritis can be diagnosed by upper gastrointestinal endoscopy, CT scan, or endoscopic ultrasound. Its endoscopic findings can show purple colored gastric mucosa covered with dirty necrotic materials. Absolute diagnosis is made most frequently at laparotomy [7]. The mortality associated with acute gastric necrosis ranges from 50 to $80 \%$ which underscores the severity or life-threatening nature of the condition. Significant mortalities have been reported in recent publications even with aggressive management. The presence of circulatory collapse may be a significant contribution to mortality [8]. The treatment of gastric ischemia includes fluid resuscitation, low intermittent nasogastric suction to combat gastric distension, intravenous proton pump inhibitor therapy, and the select usage of broadspectrum antibiotics if sepsis or gastric pneumatosis is present. Angiographic interventions may be indicated in cases of splanchnic vascular obstruction. Surgical intervention is indicated in cases of gastric perforation, gastric volvulus, or cases of severe gastric ischemia, which fail to improve with medical therapy [9]. Early resection of necrotic gastric wall combined with adequate antimicrobial therapy is the cornerstone in treatment of necrotizing gastritis [10].

\section{Declarations}

Ethical Approval Not required

Conflict of Interest The authors declare no competing interests.

\section{References}

1. Miller AI, Smith B, Rogers AI (1975) Phlegmonous gastritis. Gastroenterology. 68(2):231-238

2. Strauss RJ, Friedman M, Platt N, Gassner W, Wise L (1978) Gangrene of the stomach: a case of acute necrotizing gastritis. Am J Surg 135(2):253-257. https://doi.org/10.1016/00029610(78)90111-3

3. Zvizdic Z, Jonuzi A, Djuran A, Vranic S (2019) Gastric necrosis and perforation following massive gastric dilatation in an adolescent girl: a rare cause of acute abdomen. Front Surg 6:3. https://doi. org/10.3389/fsurg.2019.00003 
4. Salroo N, Ahangar S (2012) Gastric gangrene "an iatrogenic misadventure". Indian J Surg 74(6):498-500. https://doi.org/10.1007/ s12262-012-0509-5

5. Perigela HC, Vasamsetty MK, Bangi VP, Nagabhushigari S (2014) Gastric gangrene due to acute necrotizing gastritis. J NTR Univ Health Sci [serial online] [cited 2021 Mar 6];3:38-40. Available from: https://www.jdrntruhs.org/text.asp?2014/3/1/38/128429

6. Tang SJ, Daram SR, Wu R, Bhaijee F (2014) Pathogenesis, diagnosis, and management of gastric ischemia. Clin Gastroenterol Hepatol 12(2):246-52.e1. https://doi.org/10.1016/j.cgh.2013.07. 025

7. Tejas AP, Rajagopalan S, Rohit K (2017) Necrotizing gastritis: a case report. Int Surg J 4:3535-3538

8. Yorke J, Gyamfi FE, Awoonor-Williams R, Osei-Akoto E, Acheampong E, Acheampong EN, Adinku MO, Yamoah FA,
Konney TO, Adjei E, Boateng EA, Dally CK, Ababio KA, AffulYorke D, Ahulu D (2020) Acute gastric necrosis in a teenager. Case Rep Surg 2020:Article ID 8882179, 4 pages. https://doi.org/10. $1155 / 2020 / 8882179$

9. Sharma A, Mukewar S, Chari ST, Kee Song LMW (2017) Clinical features and outcomes of gastric ischemia. Dig Dis Sci 62:3550-3556

10. Kobus C, van den Broek JJ, Richir MC (2020) Acute gastric necrosis caused by a $\beta$-hemolytic streptococcus infection: a case report and review of the literature. Acta Chir Belg 120(1):53-56. https:// doi.org/10.1080/00015458.2018.1500799

Publisher's Note Springer Nature remains neutral with regard to jurisdictional claims in published maps and institutional affiliations. 